\title{
Scanning tunneling microscopy-from birth to adolescence
}

\author{
Gerd Binnig and Heinrich Rohrer \\ IBM Research Division, Zürich Research Laboratory, 8803 Rüschlikon, Switzerland
}

We present here the historic development of Scanning Tunneling Microscopy (STM); the physical and technical aspects have already been covered in a few recent reviews and two conference proceedings ${ }^{1}$ and many others are expected to follow in the near future. A technical summary is given by the sequence of figures, which stands alone. Our narrative is by no means a recommendation of how research should be done; it simply reflects what we thought, how we acted, and what we felt. However, it would certainly be gratifying if it encouraged a more relaxed attitude towards doing science.

Perhaps we were fortunate in having common training in superconductivity, a field which radiates beauty and elegance. For scanning tunneling microscopy, we brought along some experience in tunneling (Binnig and Hoenig, 1978) and angstroms (Rohrer, 1960), but none in microscopy or surface science. This probably gave us the courage and lightheartedness to start something which should "not have worked in principle," as we were so often told.

"After having worked a couple of years in the area of phase transitions and critical phenomena, and many, many years with magnetic fields, I was ready for a change. Tunneling, in one form or another, had intrigued me for quite some time. [See Fig. 1.] Years back, I had become interested in an idea of John Slonczewski to read magnetic bubbles with tunneling; on another occasion, I had been involved for a short time with tunneling between very small metallic grains in bistable resistors, and later I watched my colleagues struggle with tolerance problems in the fabrication of Josephson junctions. So the local study of growth and electrical properties of thin insulating layers appeared to me an interesting problem, and I was given the opportunity to hire a new research staff member, Gerd Binnig, who found it interesting, too, and accepted the offer. Incidentally, Gerd and I would have missed each other, had it not been for K. Alex Müller,

\footnotetext{
${ }^{*}$ This lecture was delivered 8 December, 1986, on the occasion of the presentation of the 1986 Nobel Prize in Physics.

${ }^{1}$ For reviews, see Binnig and Rohrer (1986); Golovchenko (1986); Behm and Hoesler (1986); Hansma and Tersoff (1987); IBM Europe (1986, Proceedings of the STM Workshop in Oberlech, Austria); and Gatos (1987, Proceedings of the First International Conference on STM, Santiago de Compostela, Spain). An article combining technical and biographical details is presented in Dordick (1986).

${ }^{2}$ H. Rohrer, quoted from Binnig and Rohrer (1986), p. 369.
}

then head of Physics, who made the first contacts."2

The original idea then was not to build a microscope but rather to perform spectroscopy locally on an area less than $100 \AA$ in diameter.

"On a house-hunting expedition, three months before my actual start at IBM, Heini Rohrer discussed with me in more detail his thoughts on inhomogeneities on surfaces, especially those of thin oxide layers grown on metal surfaces. Our discussion revolved around the idea of how to study these films locally, but we realized that an appropriate tool was lacking. We were also puzzling over whether arranging tunneling contacts in a specific manner would give more insight on the subject. As a result of that discussion, and quite out of the blue at the LT15 Conference in Grenoble-still some weeks before I actually started at IBM-an old dream of mine stirred at the back of my mind, namely, that of vacuum tunneling. I did not learn until several years later that I had shared this dream with many other scientists, who like myself, were working on tunneling spectroscopy. Strangely enough, none of us had ever talked about it, although the idea was old in principle." 3 Actually, it was 20 years old, dating back to the very beginning of tunneling spectroscopy (Giaever, 1974). Apparently, it had mostly remained an idea, and only shortly after we had started, did Seymour Keller, then a member of the IBM Research Division's Technical Review Board and an early advocate of tunneling as a new research area in our Laboratory, draw our attention to W. A. Thompson's attempting vacuum tunneling with a positionable tip (Thompson and Hanrahan, 1976). (See Fig. 2.)

We became very excited about this experimental challenge and the opening up of new possibilities. Astonishingly, it took us a couple of weeks to realize that not only would we have a local spectroscopic probe, but that scanning would deliver spectroscopic and even topographic images, i.e., a new type of microscope. The operating mode mostly resembled that of stylus profilometry (Williamson, 1967; Guenther et al., 1984), but instead of scanning a tip in mechanical contact over a surface, a small gap of a few angstroms between tip and sample was maintained and controlled by the tunnel current flowing between them. Roughly two years later and shortly before getting our first images, we learned about a paper by $\mathbf{R}$. Young et al. (1972) where they described a type of fieldemission microscope they called "topografiner." It had

${ }^{3}$ G. Binnig, quoted from Binnig and Rohrer (1986), p. 369. 
(a)

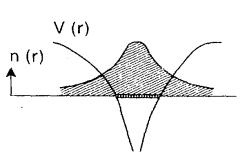

(b)

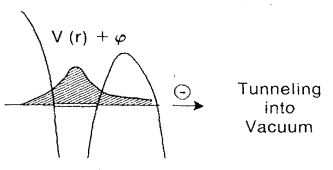

(c)

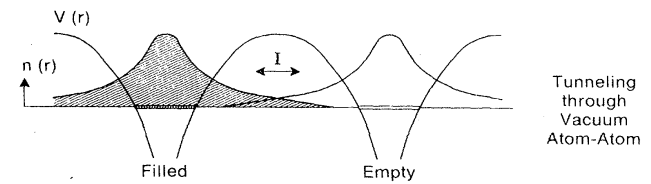

(d)

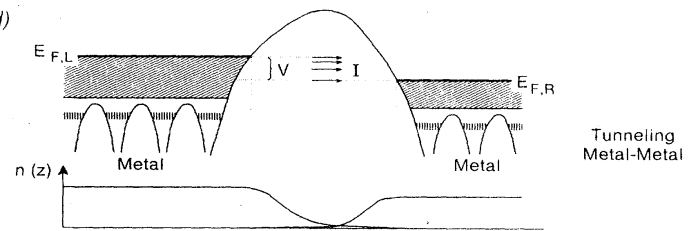

(e)

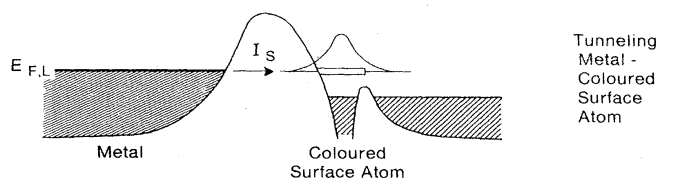

FIG. 1. Tunneling. (a) The wave function of a valence electron in the Coulomb potential well of the atom core plus other valence electrons extends into the vacuum; it "tunnels" into the vacuum. (b) Exposed to an electric field the electron can tunnel through the potential barrier and leave the atom. (c) If two atoms come sufficiently close, then an electron can tunnel back and forth through the vacuum or potential barrier between them. (d) In a metal, the potential barriers between the atoms in the interior are quenched and electrons move freely in energy bands, the conduction bands. At the surface, however, the potential rises on the vacuum side forming the tunnel barrier, through which an electron can tunnel to the surface atom of another metal close by. The voltage $V$ applied between the two metals produces a difference between the Fermi levels $E_{F, L}$ and $E_{F, R}$, thus providing empty states on the right for the electrons tunneling from the left side. The resulting tunnel current is roughly of the form $I=f(V) \exp (-\sqrt{\bar{\phi}} \cdot s)$. The $f(V)$ contains a weighted joint local density of states of tip and object, the exponential gives the transmittivity with $\bar{\phi}$ the averaged tunnel barrier height in $\mathrm{eV}$, and $s$ the separation of the two metals in $\AA$. Here $f(V)$ and $\sqrt{\bar{\phi}}$ are material properties obtained by measuring $d \ln I / d V$ and $d \ln I / d s$. (e) A simple case of local spectroscopy. A characteristic state, the "color," of a surface species, is observed by the onset of the tunnel-current contribution $l_{s}$ [see Lang (1987) and references therein].

much in common with our basic principle of operating the STM, except that the tip had to be rather far away from the surface, thus on high voltage producing a fieldemission current rather than a tunneling current and resulting in a lateral resolution roughly that of an optical microscope. They suggested to improve the resolution by using sharper field-emission tips, even attempted vacuum tunneling, and discussed some of its exciting prospects in spectroscopy. Had they, even if only in their minds, com-
Oxide Junction
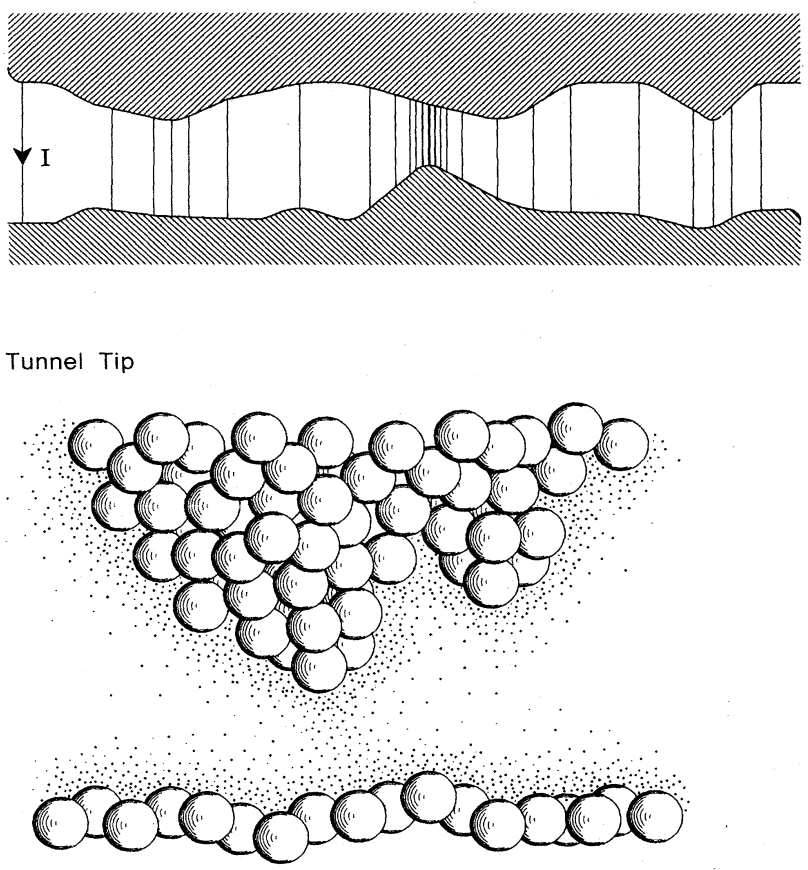

FIG. 2. The principle. The tunneling transmittivity decreases exponentially with the tunneling distance, in vacuum about a factor of 10 for every $\AA$. In an oxide tunnel junction, most of the current flows through narrow channels of small electrode separation. With one electrode shaped into a tip, the current flows practically only from the front atoms of the tip, in the best case from a specific orbital of the apex atom. This gives a tunnel current filament width and thus a lateral resolution of atomic dimensions. The second tip shown is recessed by about two atoms and carries about a million times less current.

bined vacuum tunneling with scanning, and estimated that resolution, they would probably have ended up with the new concept, Scanning Tunneling Microscopy. They came closer than anyone else.

Mid-January 1979, we submitted our first patent disclosure on STM. Eric Courtens, then deputy manager of physics at the IBM Rüschlikon Laboratory, pushed the disclosure to a patent application with "thousands of future STM's." $\mathrm{He}$ was the first believer in our cause. Shortly afterwards, following an in-house seminar on our STM ideas, Hans-Jörg Scheel became the third.

For the technical realization of our project, we were fortunate in securing the craftsmanship of Christoph Gerber. "Since his joining IBM in 1966, Christoph had worked with me (HR) on pulsed high-magnetic fields, on phase diagrams, and on critical phenomena. By the end of 1978, we were quite excited about our first experimental results on the random-field problem, but when asked to participate in the new venture, Christoph did not hesitate an instant. He always liked things which were out of the ordinary, and, incidentally, was the second believer. This left me and the random-field problem without his diligent technical support. About a year later, Edi Weibel 


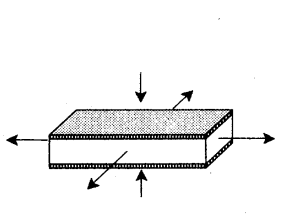

(a)

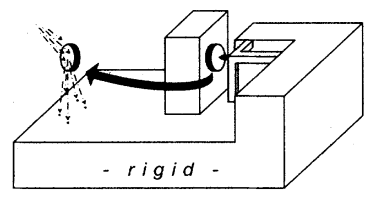

(d)

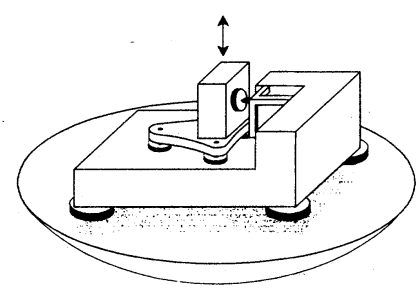

(f) (b)

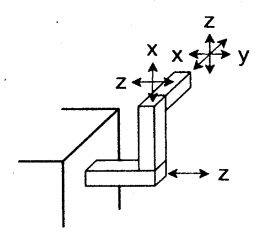

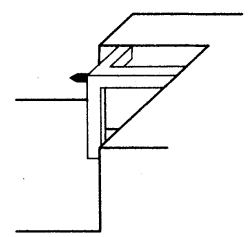

(c)

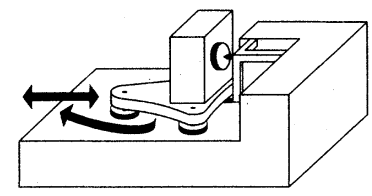

(e)

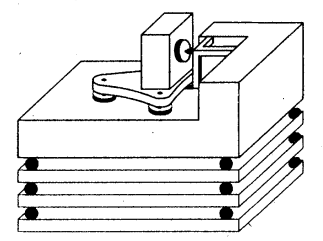

(g)
FIG. 3. The instrument. (a) A voltage applied to two electrodes contracts or expands the piezoelectric material in between. The practical total excursion of a piezo is usually in the region of micrometers. (b) A frictionless $x-y-z$ piezodrive, which is quite vibration sensitive. (c) A rigid tripod is at present the piezodrive most used apart from the single-tube scanner. (d) Tripod and sample holder are installed on a rigid frame. The sample has to be cleared from the tip for preparation and sample transfer. (e) Positioning of the sample to within reach of the piezodrive was originally achieved with a piezoelectric "louse" with electrostatically clampable feet. Magnetic-driven positioners and differential screws are also now in use. (f) In the first vibrationisolation system, the tunnel unit with permanent magnets levitated on a superconducting lead bowl. (g) The simple and presently widely used vibration protection with a stack of metal plates separated by viton-a UHV-compatible rubber spacer.

was the next one to join in, which left another project without technical support. Finally, I completed the team, leaving the random-field problem to others." 4

During the first few months of our work on the STM, we concentrated on the main instrumental problems and their solutions (Binnig and Rohrer, 1982, 1983, 1985): How to avoid mechanical vibrations that move tip and sample against each other? Protection against vibrations and acoustical noise by soft suspension of the microscope within a vacuum chamber. How strong are the forces between tip and sample? This seemed to be no problem in most cases. How to move a tip on such a fine scale? With piezoelectric material, the link between electronics

${ }^{4}$ H. Rohrer, quoted from Dordick (1986), p. 4.
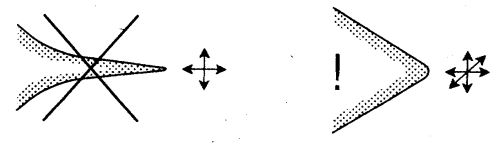

(b)
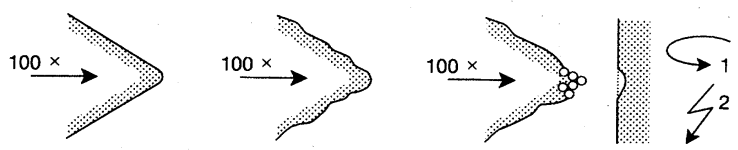

(c)

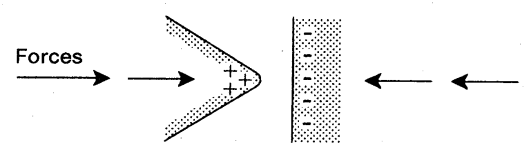

FIG. 4. Tips. (a) Long and narrow tips, or whiskers, are vibration sensitive and thermally excited. (b) A mechanically ground or etched tip shows sharp minitips, only one of which usually carries the tunnel current. Further sharpening was initially achieved with gentle contact (1), later with field evaporation (2). (c) Electrostatic and interatomic forces between tip and sample do not deform a blunt tip, or a rigid sample, but they make the tunnel gap mechanically unstable when the tip carries a whisker. The responses of soft sample materials like graphite or organic matter to such forces, however, can be appreciable and have to be taken into account.

and mechanics, avoiding friction. The continuous deformation of piezomaterial in the angstrom and subangstrom range was established only later by the tunneling experiments themselves. How to move the sample on a fine scale over long distances from the position of surface treatment to within reach of the tip? (See Fig. 3.) The "louse." How to avoid strong thermally excited length fluctuations of the sample and especially the tip? Avoid whiskers with small spring constants. This led to a more general question, and the most important one: What should be the shape of the tip and how to achieve it? At the very beginning, we viewed the tip as a kind of continuous matter with some radius of curvature. However, we very soon realized that a tip is never smooth because of the finite size of atoms and because tips are quite rough unless treated in a special way. This roughness implies the existence of minitips as we called them, and the extreme sensitivity of the tunnel current on tip-sample separation then selects the minitip reaching closest to the sample. (See Fig. 4.)

Immediately after having obtained the first stable STM images showing remarkably sharp monoatomic steps, we focused our attention on atomic resolution. Our hopes of achieving this goal were raised by the fact that vacuum tunneling itself provides a new tool for fabricating extremely sharp tips: The very local, high fields obtainable with vacuum tunneling at a few volts only can be used to shape the tip by field migration or by field evaporation: Gently touching the surface is another possibility. All this is not such a controlled procedure as tip sharpening in field-ion microscopy, but it appeared to us to be too 
complicated to combine STM with field-ion microscopy at this stage. We hardly knew what field-ion microscopy was, to say nothing of working with it. We had no means of controlling exactly the detailed shape of the tip. We repeated our trial and error procedures until the structures we observed became sharper and sharper. Sometimes it worked, other times it did not.

But first we had to demonstrate vacuum tunneling. In this endeavor, apart from the occurrence of whiskers, the most severe problem was building vibrations. To protect the STM unit also against acoustical noise, we installed the vibration-isolation system within the vacuum chamber. Our first setup was designed to work at low temperatures and in ultrahigh vacuum (UHV). Low temperatures guaranteed low thermal drifts and low thermal length fluctuations, but we had opted for them mainly because our thoughts were fixed on spectroscopy. And tunneling spectroscopy was a low-temperature domain for both of us with a Ph.D. education in superconductivity. The UHV would allow preparation and retention of welldefined surfaces. The instrument was beautifully designed with sample and tip accessible for surface treatments and superconducting levitation of the tunneling unit for vibration isolation. Construction and first lowtemperature and UHV tests took a year, but the instrument was so complicated, we never used it. We had been too ambitious, and it was only seven years later that the principal problems of a low-temperature and UHV instrument were solved (Marti, 1986; Marti et al., 1987). Instead, we used an exsiccator as vacuum chamber, lots of Scotch tape, and a primitive version of superconducting levitation wasting about 201 of liquid helium per hour. Emil Haupt, our expert glass blower, helped with lots of glassware and, in his enthusiasm, even made the lead bowl for the levitation. Measuring at night and hardly daring to breathe from excitement, but mainly to avoid vibrations, we obtained our first clear-cut exponential dependence of the tunnel current $I$ on tip-sample separation $s$ characteristic for tunneling. (See Fig. 5.) It was the portentous night of 16 March, 1981.

So, 27 months after its conception the Scanning Tunneling Microscope was born. During this development period, we created and were granted the necessary elbowroom to dream, to explore, and to make and correct mistakes. We did not require extra manpower or funding, and our side activities produced acceptable and publishable results. The first document on STM was the March/April 1981 in-house Activity Report.

A logarithmic dependence of the tunnel current $I$ on tip-sample separation $s$ alone was not yet proof of vacuum tunneling. The slope of $\ln I$ vs $s$ should correspond to a tunnel-barrier height of $\phi \approx 5 \mathrm{eV}$, characteristic of the average work functions of tip and sample. We hardly arrived at $1 \mathrm{eV}$, indicating tunneling through some insulating material rather than through vacuum. Fortunately, the calibration of the piezosensitivity for small and fast voltage changes gave values only half of those quoted by the manufacturers. This yielded a tunnel-barrier height of more than $4 \mathrm{eV}$ and thus established vacuum tunneling.
Constant Current Mode

(a)

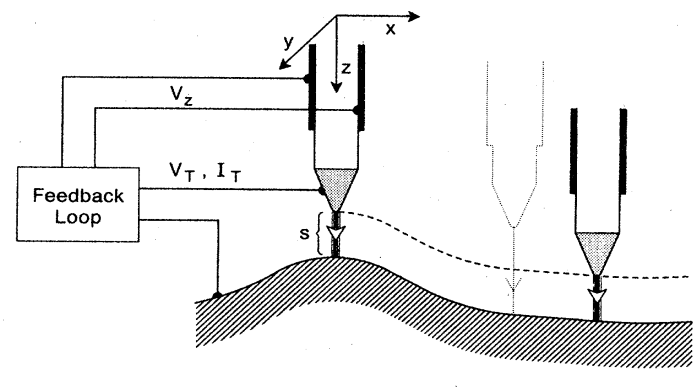

$v_{z}\left(v_{x}, v_{y}\right) \rightarrow z(x, y)$

(b)

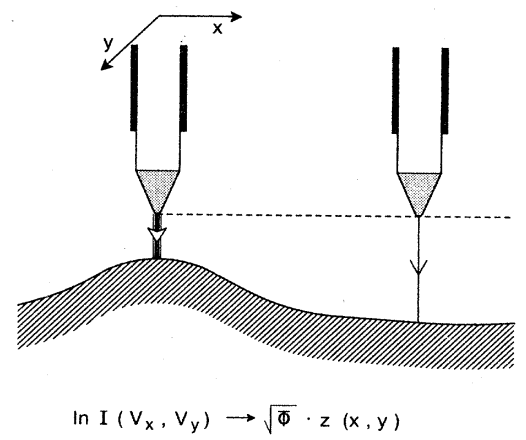

FIG. 5. Imaging. (a) In the constant current mode, the tip is scanned across the surface at constant tunnel current, maintained at a preset value by continuously adjusting the vertical tip position with the feedback voltage $V_{z}$. In the case of an electronically homogeneous surface, constant current essentially means constant $s$. (b) On surface portions with denivellations less than a few $\AA$-corresponding to the dynamic range of the current measurement-the tip can be rapidly scanned at constant average $z$ position. Such "current images" allow much faster scanning than in (a) but require a separate determination of $\sqrt{\phi}$ to calibrate $z$. In both cases, the tunnel voltage and/or the $z$ position can be modulated to obtain, in addition, $d \ln I / d V$ and/or $d \ln I / d s$, respectively.

This reduced piezosensitivity was later confirmed by careful calibration with $\mathbf{H}$. R. Ott from ETH, Zürich, and S. Vieira of the Universidad Autónoma, Madrid (Ott and Rohrer, 1981; Vieira, 1986).

U. Poppe had reported vacuum tunneling some months earlier (Poppe, 1981), but his interest was tunneling spectroscopy on exotic superconductors. He was quite successful at that but did not measure $I(s)$. Eighteen months later, we were informed that E. C. Teague, in his thesis, had already observed similar $I(s)$ curves which at that time were not commonly available in the open literature (Teague, 1978a, 1978b, 1986).

Our excitement after that March night was quite considerable. Hirsh Cohen, then Deputy Director of our Laboratory, immediately asked us "what do you need?", a simple and obvious question people only rarely dare to ask. "Gerd immediately wanted to submit a postdeadline contribution (Binnig et al., 1982a) to the LT16 Conference to be held in Los Angeles in September. He was go- 
ing there anyway with his superconducting strontium titanate, and I was sure we would have some topographic STM images by then. And indeed we had. I arranged an extended colloquium tour through the USA for Gerd, but about three weeks before his departure, a friend warned him that, once the news became public, hundreds of scientists would immediately jump onto the STM bandwagon. They did-a couple of years later. After two extended discussions on a weekend hike, he nevertheless became convinced that it was time for the STM to make its public appearance." Our first attempt to publish a letter failed. "That's a good sign," Nico Garcia, a Visiting Professor from the Universidad Autónoma de Madrid, Spain, consoled us.

After this first important step with a complete STM setup, it took us only three months, partly spent waiting for the high-voltage power supplies for the piezos, to obtain the first images of monosteps (Binnig et al., 1982b) on a CaIrSn 4 single crystal grown by $\mathrm{R}$. Gambino. Here, the main problem was getting rid of the whiskers we continually created by bumping the tip into the surface. Now we were ready to turn to surface science, first to resolve surface reconstructions. We built a UHV-compatible STM (no longer with Scotch tape!) and as a quick trial, operated it in vacuum suspended from a rubber band. The results indicated that superconducting levitation might be unnecessary.

That was the state of the art for the publicity tour through the USA in September '81. Most reactions were benevolent, some enthusiastic, and two even anticipated the Nobel prize, but the STM was apparently still too exotic for any active outside engagement.

Next, we protected the STM from vibrations by a double-stage spring system with eddy-current damping (Binnig and Rohrer, 1982, 1983, 1985), and incorporated it in a UHV chamber not in use at that moment. We added sputtering and annealing for sample treatment, but no other surface tool to characterize and monitor the state of the sample or tip could yet be combined with that STM. Although the superconducting levitation served for three months only, it was cited for years. It would appear that something complicated is much easier to remember!

A most intriguing and challenging surface-science problem existed, namely, the $7 \times 7$ reconstruction of the $\mathrm{Si}(111)$ surface. A class of fashionable models contained rather rough features which should be resolvable by the STM. So we started to chase after the $7 \times 7$ structure, and succumbed to its magic. At first, with no success. The STM would function well, sometimes with resolutions clearly around $5 \AA$, but not our surface preparation. We occasionally found quite nice patterns with monolayer step lines (Binnig and Rohrer, 1982, 1983, 1985), but usually the surface looked rough and disordered on an atomic scale. One image even foreshadowed the $7 \times 7$ by a regular pattern of depressions, the precursors of the characteristic corner holes. However, a single event is too risky to make a case for a new structure obtained with a new method. But it boosted our confidence.

By spring '82, STM was already a subject talked about.
Supposedly, an image of a vicinal surface expertly prepared with a regular step sequence would have eased the somewhat reserved attitude of the surface-science community. We, however, thought that the mono, double, and triple steps of the CaIrSn ${ }_{4}$ with atomically flat terraces (Binnig et al., 1982b) and the step lines of $\mathrm{Si}(111)$ (Binnig and Rohrer, 1982, 1983, 1985) were convincing and promising enough. And instead of wasting further time on uninteresting step lines, we preferred to attack surface reconstructions with known periodicities and with a reasonable chance of learning and contributing something new.

For easier sample preparation and because the demand on resolution was only $8 \AA$, we changed to a gold single crystal, namely, the (110) surface known to produce a $1 \times 2$ reconstruction. This seemed to be well within reach of the STM resolution from what we had learned from the silicon step lines. Although some time earlier we had returned to Karl-Heinz Rieder, the Laboratory's surfacescience expert, his $\mathrm{Si}$ single crystal in a kind of droplet form, it did not deter him from proposing this gold experiment which meant lending us his Au crystal, and some weeks later we added another droplet to his collection! But in between, with his advice on surface preparation, we succeeded in resolving the $1 \times 2$ structure (Binnig et al., 1983a). Contrary to expectations, we also had to struggle with resolution, because Au transferred from the surface even if we only touched it gently with our tip. The mobility of $\mathrm{Au}$ at room temperature is so high that rough surfaces smooth out after a while, i.e., really sharp Au-coated tips cease to exist. We should like to mention here that later, for measurements on $\mathrm{Au}(100)$, we formed sharp $\mathrm{Au}$ tips by field evaporation of $\mathrm{Au}$ atoms from sample to tip, and could stabilize them by a relatively high field resulting from a $0.8-\mathrm{V}$ tunnel voltage.

In the case of the $\mathrm{Au}(110)$ surface, the atomic resolution was rather a matter of good luck and perseverance. It jumped from high to low in an unpredictable manner, which was probably caused by migrating adatoms on the tip finding a stable position at the apex for a while. We also observed an appreciable disorder leading to long but narrow ribbons of the $1 \times 2$ reconstruction mixed with ribbons of $1 \times 3$ and $1 \times 4$ reconstructions and step lines. Nevertheless, these experiments were the first STM images showing atomic rows with atomic resolution perpendicular to the rows. The disorder, intrinsic on this surface, but in its extent criticized from the surface-science point of view, demonstrated very nicely the power of STM as a local method, and about a year later played an important role in testing the first microscopic theories of scanning tunneling microscopy.

With gold, we also performed the first spectroscopy experiment with an STM. We wanted to test a prediction regarding the rectifying $I-V$ characteristic of a sample-tip tunnel junction induced by the geometric asymmetry (Miskowsky et al., 1980). Unfortunately, the sample surface became unstable at around $5 \mathrm{~V}$, sample positive, and the small asymmetry observed in this voltage range could also have been due to other reasons. But with reversed 
polarity, the voltage could be swept up to $20 \mathrm{~V}$, producing a whole series of marked resonant surface states (Binnig and Rohrer, 1982, 1983, 1985). We consider the gold exercise during spring and early summer of ' 82 a most important step in the development of the method, and the STM had already exceeded our initial expectations. We had also won our first believers outside the Laboratory, Cal Quate from Stanford University (Quate, 1986) and Paul Hansma from the University of California at Santa Barbara (Moreland et al., 1983). ${ }^{5}$ We gave numerous talks on the Au work, and it attracted some attention, but all in all there was little action. We did not even take the time to write a paper-the $7 \times 7$ was waiting!

Meanwhile, we had also made the first attempts at chemical imaging: small Au islands on silicon. The islands were visible as smooth, flat hills on a rough surface in the topography, but they were also clearly recognizable as regions with enhanced tunnel-barrier height (Binnig and Rohrer, 1982, 1983, 1985). Thus the Au islands were imaged thanks to their different surface electronic properties. It would certainly have been interesting to pursue this line, but we knew that, in principle, it worked-and the $7 \times 7$ was still waiting!

We started the second $7 \times 7$ attempt in autumn 1982 , taking into consideration the advice of Franz Himpsel not to sputter the surface. This immediately worked and we observed the $7 \times 7$ whenever the surface was flat. We were absolutely enchanted by the beauty of the pattern.

"I could not stop looking at the images. It was like entering a new world. This appeared to me as the unsurpassable highlight of my scientific career and therefore, in a way, its end. Heini realized my mood and whisked me away for some days to St. Antönien, a charming village high up in the Swiss mountains, where we wrote the paper on the $7 \times 7 ., " 6$ (See Fig. 6.)

We returned convinced that this would attract the attention of our colleagues, even of those not involved with surface science. We helped by presenting both an unprocessed relief model assembled from the original recorder traces with scissors, Plexiglas, and nails, and a processed top view; the former for credibility, the latter for analysis and discussion (Binnig et al., 1983b). It certainly did help, with the result that we practically stopped doing research for a while. We were inundated with requests for talks, and innumerable visitors to our laboratory were curious to know how to build an STM. However, the number of groups that seriously got started remained small. It seemed there was still a conflict between the very appealing, conceptual easiness of displaying individual atoms in three-dimensional real space direct by recorder traces, and the intuitive reservation that, after

\footnotetext{
${ }^{5}$ Actually, Paul Hansma was indisposed and could not attend the first seminar given on STM in the USA. However, his students attended, and with them Paul built the squeezable tunnel junction.
}

${ }^{6} \mathrm{G}$. Binnig, quoted from private communication (1986). all, it just could not be that simple.

Our result excluded all the numerous models that existed, and strangely enough also some that followed. Only one came very close: the adatom model by $\mathrm{W}$. Harrison (1976) with just the number of adatoms not quite right. Nowadays, a variation of the adatom model, where deeper layers are also reconstructed besides the characteristic $7 \times 7$ adatom pattern (Takayanagi et al., 1985), is generally accepted and compatible with most results obtained by various experimental methods like ion channeling (Tromp and van Loenen, 1985), transmission electron diffraction (Tromp, 1985, and references therein), and more detailed STM results from other groups (Becker, Golovchenko, McRae, and Swartzentruber, 1985; Hamers et al., 1986).

The $7 \times 7$ experiments also accelerated the first theoretical efforts of STM on a microscopic level. Tersoff and Hamann (1983) and Baratoff (1984) applied Bardeen's transfer Hamiltonian formalism to the small geometries of tip and an atomically corrugated surface. García, Ocal, and Flores (1983) and Stoll, Baratoff, Selloni, and Carnevali (1984) worked out a scattering approach. The two approaches converged; they consoled us by roughly confirming our intuitive view on tunneling in small geometries by simply scaling down planar tunneling, and they certainly improved the acceptance of STM in physics circles. The theoretical treatments concentrated on the nonplanar aspect of tunneling of free electrons, and the STM results on Au(110), still unpublished, served as a testing ground. They remained unpublished for quite some time, since the flashy images of the $7 \times 7$ silicon surface somehow overshadowed the earlier $\mathrm{Au}(110)$ experiments. One reaction to the first attempt to publish them was ". . . The paper is virtually devoid of conceptual discussion let alone conceptual novelty ... I am interested in the behaviour of the surface structure of gold and the other metals in the paper. Why should I be excited about the results in this paper? . . .." It was certainly bad publication management on our part, but we were not sufficiently familiar with a type of refereeing which searches for weak points, innocently ignoring the essence.

The gold and silicon experiments showed that STM in surface science would benefit greatly from additional, in situ surface characterization, in particular low-energy electron diffraction (LEED). We had already learned that surfaces, even elaborately prepared, were frequently not as uniform and flat as generally assumed. The in situ combination of LEED with STM proved extremely helpful, avoiding searching when there was nothing to be searched, and it gave us the opportunity to learn about and work with LEED and Auger electron spectroscopy (AES). The combination of STM with other established surface-science techniques also settled a concern frequently mentioned: how much did our STM images really have in common with surfaces characterized otherwise? We did not share this concern to such a degree, as we had also learned that reconstructions extended unchanged to the immediate vicinity of defect areas, and because we could detect most contaminants or defects individually. Thus, for us, the combined instrumentation was more a practi- 


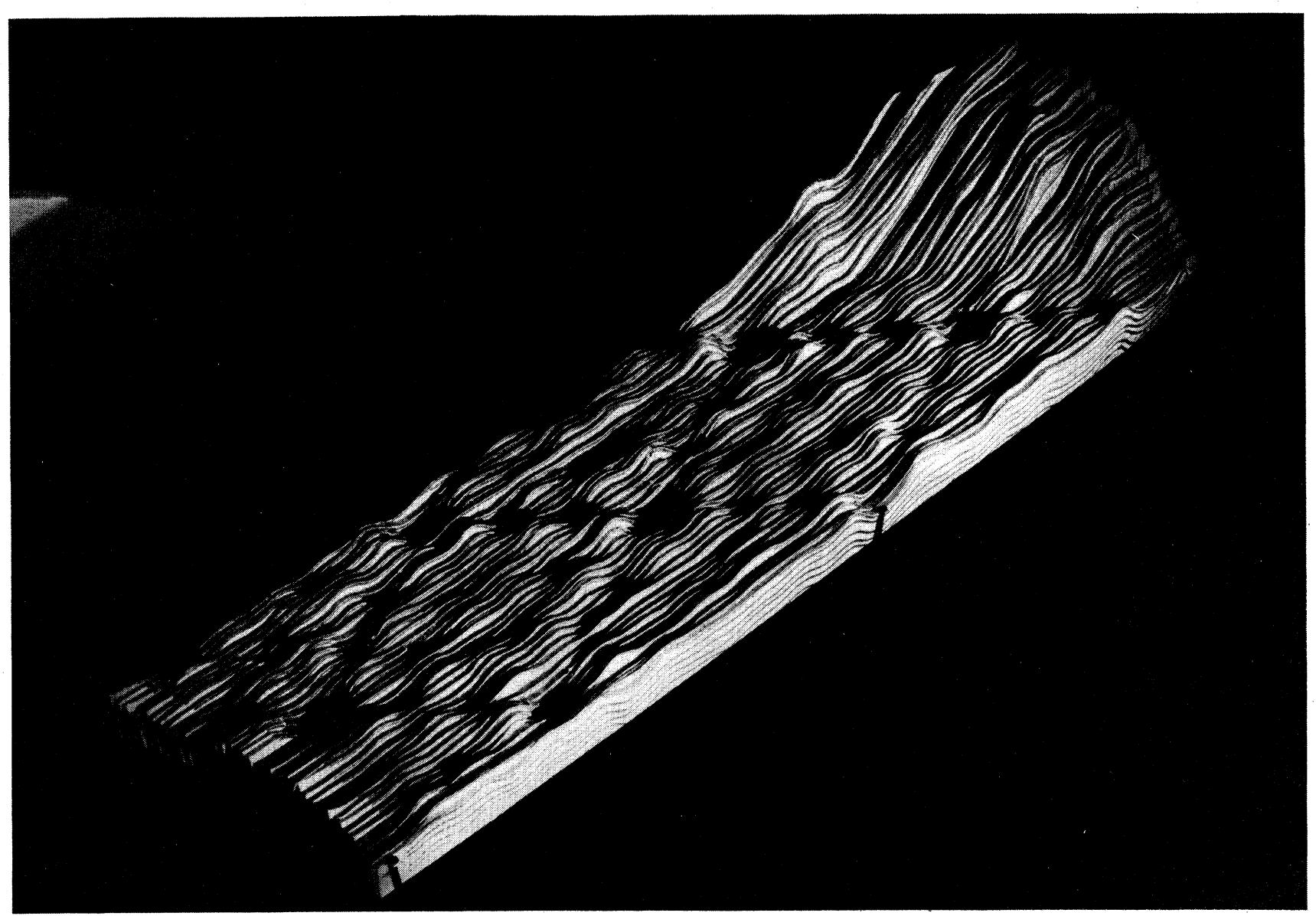

(a)

FIG. 6. $7 \times 7$ reconstruction of $\mathrm{Si}(111)$. (a) Relief assembled from the original recorder traces, from Binnig et al. (1983b), (c) 1983 The American Physical Society, and (b) processed image of the $7 \times 7$ reconstruction of Si(111). Characteristic of the rhombohedral surface unit cell are the corner hole and the 12 maxima, the adatoms. In the processed image, the six adatoms in the right half of the rhombi appear higher. This is an electronic inequivalence on the surface owing to a structural left-right inequivalence in the underlying layers. The reconstruction extends undisturbed to the immediate vicinity of the large "atom hill" on the right.

cal than a scientific issue.

After a short but interesting excursion with the new STM/LEED/AES combination into resolving and understanding the (100) surface of Au (Binnig et al.; 1984), we proceeded into the realms of chemistry. Together with A. Baró, a Visiting Professor from Universidad Autónoma de Madrid, Spain, who also wanted to familiarize himself with the technique, we observed the oxygen-induced $2 \times 1$ reconstruction of $\mathrm{Ni}(110)$ (Baró et al., 1984), interpreting the pronounced and regularly arranged protrusions we saw as individual oxygen atoms. We had seen atomicscale features before, which could be interpreted as adsorbates or adsorbate clusters, but they were more a nuisance than a matter of interest. The oxygen on $\mathrm{Ni}$ experiments demonstrated that the oxygen overlayer was not irreversibly changed by the imaging tunnel tip. This was a most significant result with regard to observing, studying, and performing surface chemistry with an STM tip. About a year later, when studying the oxygen-induced $2 \times 2$ recon- structed $\mathrm{Ni}(100)$ surface, we observed characteristic current spikes which we could attribute to oxygen diffusing along the surface underneath the tip (Binnig, Fuchs, and Stoll, 1986). We noted that the same type of spikes had already been present in our earlier images of oxygencovered $\mathrm{Ni}(110)$, but had been discarded at that time. Not only could diffusing atoms be observed individually, but their migration could be correlated to specific surface features like step lines or bound oxygen atoms, imaged simultaneously. Towards the end of 1983, we also started to probe the possibilities of STM in biology together with H. Gross from ETH, Zürich. We could follow DNA chains lying on a carbon film deposited on a Ag-coated Si wafer (Binnig and Rohrer, 1984).

That year ended with a most pleasant surprise: on Friday, 9 December, we received a telegram from the secretary of the King Faisal Foundation, followed on Monday by a phone call from the secretary of the European Physical Society announcing the King Faisal Prize of Science 


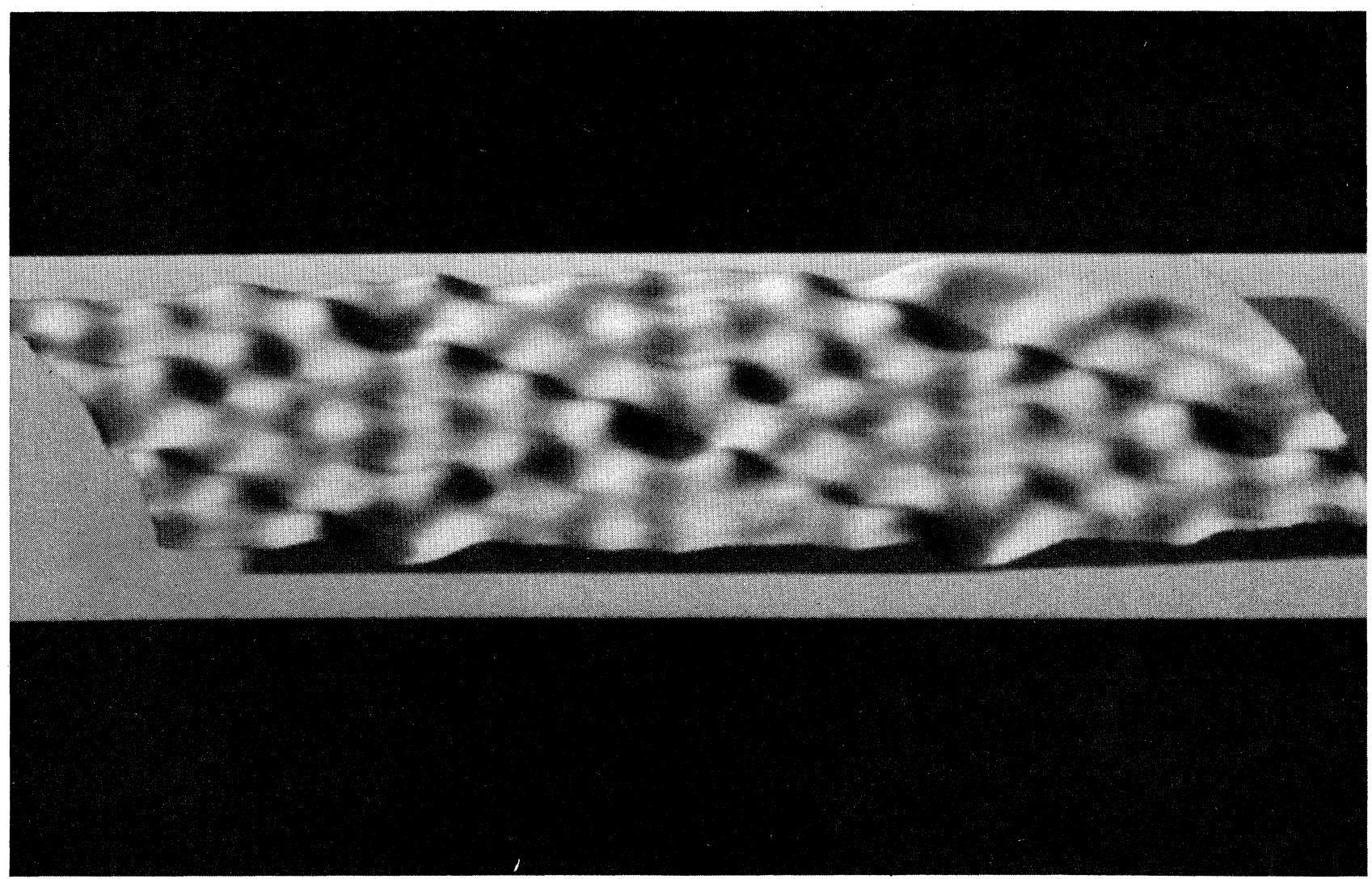

FIG. 6. (Continued).

and the Hewlett Packard Europhysics Prize, respectively. "The day the telegram arrived, Gerd was in Berlin delivering the Otto Klung Prize lecture. It was also my twentieth anniversary with IBM."7 This was an encouraging sign that Scanning Tunneling Microscopy was going to make it. It also brought a new flood of requests.

In the summer of 1984, we were finally ready to assume what we had set out to do in autumn 1978, before the notion of microscopy had ever evolved, namely, performing local spectroscopy. Together with $\mathrm{H}$. Fuchs and F. Salvan, we investigated the clean $7 \times 7$ (Baratoff et al., 1986; for reviews, see references cited in footnote 1) and the $\sqrt{3} \times \sqrt{3}$ Au reconstructions on $\mathrm{Si}(111)$ (Baratoff et al., 1986), and -right back to the heart of the matter-a thin oxide film on Ni (Binnig et al., 1985; García et al., 1986; see also reviews cited in footnote 1). We could see that surfaces are electronically structured as known, for example, from photoemission experiments, and that we could resolve these electronic structures in space on an atomic scale. We called this (and still do) the color of the atoms. Indeed, the oxide layers were inhomo-

${ }^{7}$ H. Rohrer, quoted from private communication (1986). geneous and most clearly visible in scanning tunneling spectroscopy (STS) images. On the $7 \times 7$, we could see by STS down to the second layer and observe individual dangling bonds between the adatoms (see reviews cited in footnote 1). At that time, C. Quate and his group already had an STM running, and they had performed local spectroscopy, not yet with atomic resolution but at low temperature (de Lozanne et al., 1985). They had measured the energy gap of a superconductor, and later even plotted its spatial dependence. Spectroscopic imaging was not really surprising, yet it was an important development. We now had the tools to fully characterize a surface in terms of topographic and electronic structure. Although it is usually quite an involved problem to separate the property of interest from a set of STM and STS measurements, our vision of the scanning tunneling microscope had become true. But nevertheless, we heard that this view was not generally shared. Rumors reached us that scientists would bet cases of champagne that our results were mere computer simulations! The bets were probably based on the fact the STM was already three years old, and atomic resolution was still our exclusive property. This was also our concern, but in another way. In late summer '83, Herb Budd, promoter of the IBM Europe Institute and an enthusiastic STM supporter, had asked us to run an STM seminar in summer 1984 within the 
framework of the Institute. This meant one week with 23 lectures in front of a selected audience of the European academia. At that time, there was no way whatsoever of filling 23 hours, let alone of committing 23 speakers. A year later, we agreed, full of optimism for summer '85. In December '84, on Cal Quate's initiative, nine representatives of the most advanced STM groups came together for a miniworkshop in a hotel room in Cancun. It was a most refreshing exchange of ideas, but there was still no other atomic resolution, and thus not a sufficient number of lectures in sight for the seminar.

In the following few months, the situation changed drastically. R. Feenstra and co-workers came up first with cleaved GaAs (Feenstra and Fein, 1985), C. F. Quate's group with the $1 \times 1$ structure on $\mathrm{Pt}(100)$ (Elrod et al., 1986), and J. Behm, W. Hoesler, and E. Ritter with the hexagonal phase on $\mathrm{Pt}(100)$ (Behm et al., 1986). At the American Physical Society March meeting in 1985, P. Hansma presented STM images of graphite structures of atomic dimensions (Hansma, 1985), and when J. Golovchenko unveiled the beautiful results on the various reconstructions of Ge films deposited on $\mathrm{Si}(111)$ (Becker, Golovchenko, and Swartzentruber, 1985), one could have heard a pin drop in the audience. The atomic resolution was official, and scanning tunneling microscopy accepted. The IBM Europe Institute Seminar in July turned into an exclusive workshop for STM'ers, and comprised some 35 original contributions, not all of them on atomic resolution, but already more than in March (IBM Europe, 1986). "A watershed of ideas," as Cal Quate expressed it.

Our story so far has dealt mainly with the striving for structural and electronic imaging in a surface-science environment with atomic resolution. Individual atoms had been seen before with field-ion microscopy, and dealt with individually by the atom probe technique (for a review, see Ernst and Ehrlich, 1986). The beauty of these techniques is relativized by the restriction to distinct atom sites on fine tips made from a rather limited selection of materials. Similarly, electron microscopy, the main source of present-day knowledge on submicron structures in practically all areas of science, technology, and industry, has advanced to the atomic level. Imaging of individual atoms or atomic structures, however, is still reserved for specific problems, expertise, and extraordinary equipment. The appeal and the impact of STM lie not only in the observation of surfaces atom by atom, but also in its widespread applicability, its conceptual and instrumental simplicity, and its affordability, all of which have resulted in a relaxed and almost casual perception of atoms and atomic structures. (See Fig. 7.)

But there are many other aspects, maybe less spectacular but nonetheless significant, which have made STM an accepted and viable method now pursued in many areas of science and technology.

The instruments themselves have become simpler and smaller. Their greatly reduced size allows easy incorporation into other systems, for instance, into a scanning electron microscope (Gerber et al., 1985). One type of instrument retains accurate sample positioning but is sufficient-

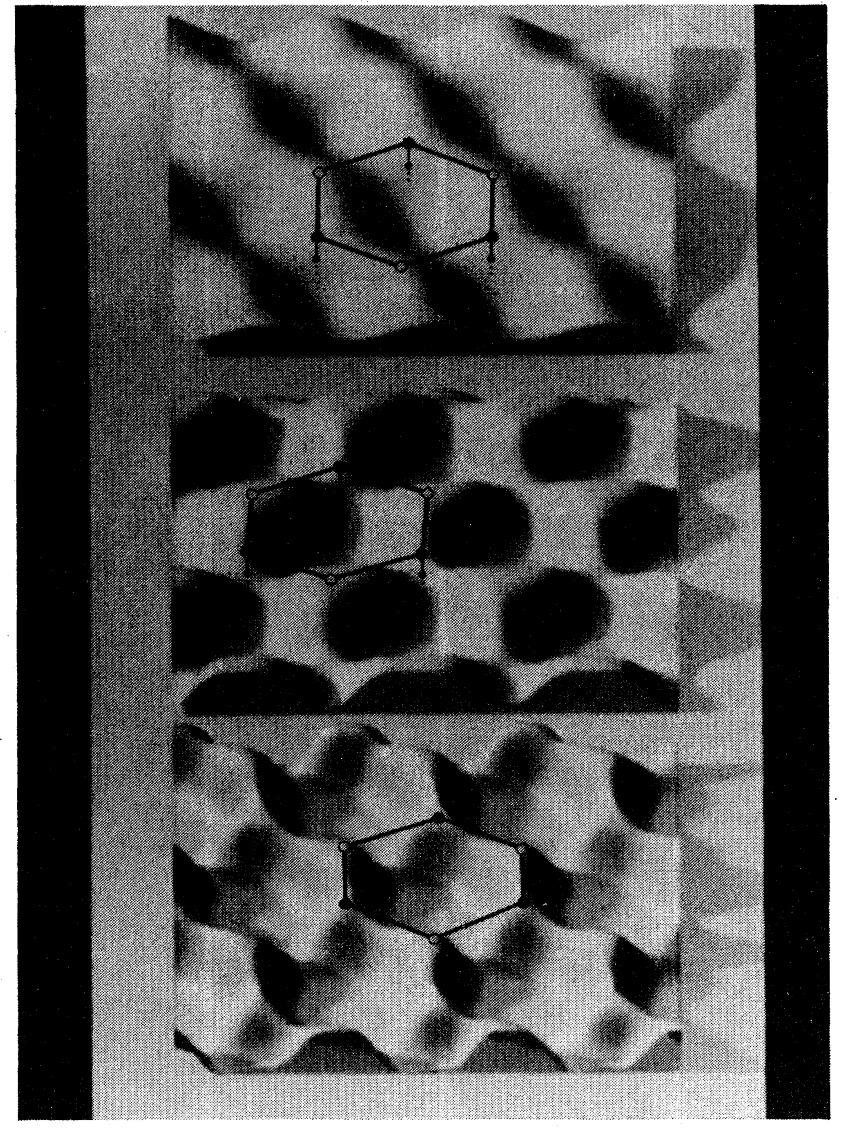

FIG. 7. STM image of cleaved graphite. The top image was taken at a constant tunnel current of $1 \mathrm{nA}$ and at $50 \mathrm{mV}$. The corrugation traced by the tip reflects the local density of states (LDOS) at the Fermi level and not the positions of atoms, which form a flat honeycomb lattice as indicated. The LDOS at the atoms bound to the neighbors in the second layer (open circles) is lower than at the "free" atoms. The image is thus rather a spectroscopic than a topographic one. The middle image is a "current image" showing essentially the same pattern. In the bottom current image, taken closer to the surface, the two inequivalent atoms appear practically identical. This peculiar behavior is compatible with a different local elastic response of the two types of carbon atoms to the interatomic force exerted by the tip compensating for their different LDOS. A local perturbation of the electronic structure might also be important.

ly rigid for in situ sample and tip exchange. Other instruments are so rigid they are even insensitive to vibrations when immersed in liquid nitrogen (Coleman et al., 1985), and even small enough to fit through the neck of a liquid-helium storage vessel (Smith and Binnig, 1986). These hummingbirds of STM, some concepts of which reach back to the squeezable tunnel junctions (Moreland et al., 1983), can also operate at television speed on relatively flat surfaces using single-tube scanners (Smith and Binnig, 1986; Bryant et al., 1986). Also tip preparation has advanced to a level where well-defined pyramidal tips 


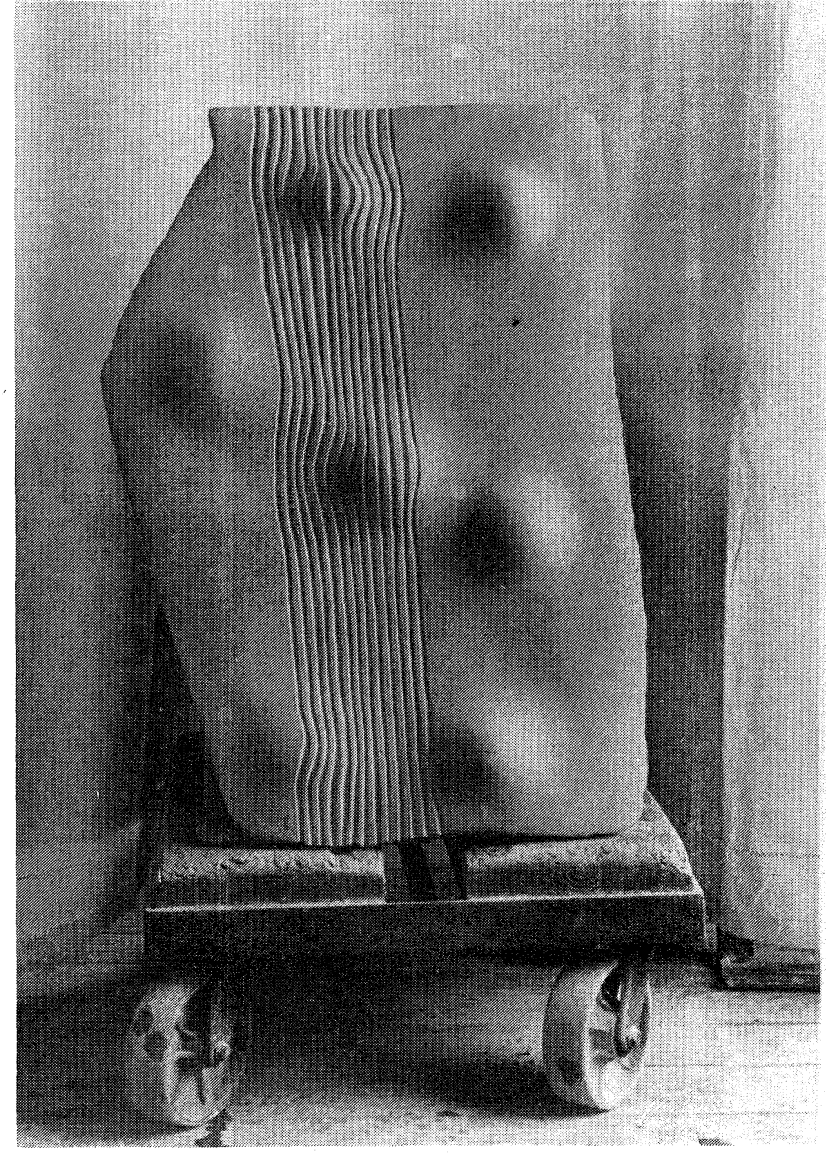

FIG. 8. Artist's conception of spheres. Art and science are both products of the creativity of man, and the beauty of nature is reflected in both. Ruedi Rempfler, the sculptor, found his interpretation in the deformation of a surface. It was the tension of the sphere in its environment which fascinated him, more than the mere portrayal of its shape. An independent creation, its visual and conceptual similarity with Fig. 6 is astounding. Original sculpture by Ruedi Rempfler, photograph courtesy of Thomas P. Frey.

ending with one (Fink, 1986) or more (Kuk and Silverman, 1986) atoms can be fabricated in a UHV environment. Such tips are particularly important for investigations of nonperiodic structures, disordered systems, and rough surfaces. They are also interesting in their own right, for example, as low-energy electron and ion point sources.

Outside the physics and surface-science communities, the various imaging environments and imaging capabilities seem as appealing as atomic resolution. Images obtained at ambient-air pressure were first reported in 1984 (Baró et al., 1985), followed by imaging in cryogenic liquids (Coleman et al., 1985), under distilled water (Sonnenfeld and Hansma, 1986), in saline solutions (Sonnenfeld and Hansma, 1986), and in electrolytes (Sonnenfeld and Schardt, 1986). Scanning tunneling potentiometry appears to have become an interesting technique to study the potential distribution on an atomic scale of currentcarrying microstructures (Muralt and Pohl, 1986). More recent advances include interatomic-force imaging with the atomic-force microscope (Binnig, Quate, and Gerber, 1986), with which the structure and elastic properties of conductors and insulators are obtained, and combined imaging of electronic and elastic properties of soft materials (Dürig et al., 1986; Soler et al., 1986). Also the use of spin-polarized electron tunneling to resolve magnetic surface structures is being explored.

Finally, we revert to the point where the STM originated: the performance of a local experiment, at a preselected position and on a very small spatial scale down to atomic dimensions. Besides imaging, it opens, quite generally, new possibilities for experimenting, whether to study nondestructively or to modify locally: local high electric fields, extreme current densities, local deformations, measurements of small forces down to those between individual atoms, just to name a few, ultimately to handle atoms (Becker et al., 1987) and to modify individual molecules, in short, to use the STM as a Feynman Machine (Feynman, 1960; Hameroff et al., 1987). This area has not yet reached adolescence.

The STM's "Years of Apprenticeship" have come to an end, the foundations have been laid, and the "Years of Travel" begin. We should not like to speculate where it will finally lead, but we sincerely trust that the beauty of atomic structures might be an inducement to apply the technique to those problems where it will be of greatest service solely to the benefit of mankind. (See Fig. 8.) Alfred Nobel's hope, our hope, everybody's hope.

We should like to thank all those who have supported us in one way or another, and those who have contributed to the development of Scanning Tunneling Microscopy, and express our appreciation of the pleasant and collegial atmosphere existing in the STM community. Thanks are also due to Dilys Brüllmann for her diligent handling of our manuscripts from the start and for her careful reading of this manuscript, and to Erich Stoll for processing Figs. 6 and 7 using ideas of R. Voss.

\section{REFERENCES}

Baratoff, A., 1984, Physica B 127, 143.

Baratoff, A., G. Binnig, H. Fuchs, F. Salvan, and E. Stoll, 1986, Surf. Sci. 168, 734.

Baró, A. M., G. Binnig, H. Rohrer, Ch. Gerber, E. Stoll, A. Baratoff, and F. Salvan, 1984, Phys. Rev. Lett. 52, 1304.

Baró, A. M., R. Miranda, J. Alamán, N. García, G. Binnig, H. Rohrer, Ch. Gerber, and J. L. Carrascosa, 1985, Nature (London) 315, 253.

Becker, R. S., J. A. Golovchenko, E. G. McRae, and B. S. Swartzentruber, 1985, Phys. Rev. Lett. 55, 2028.

Becker, R. S., J. A. Golovchenko, and B. S. Swartzentruber, 1985, Phys. Rev. Lett. 54, 2678.

Becker, R. S., J. A. Golovchenko, and B. S. Swartzentruber, 
1987, Nature (London) 325, 419.

Behm, R. J., and W. Hoesler, 1986, Physics and Chemistry of Solid Surfaces (Springer, Berlin), Vol. VI, p. 361.

Behm, R. J., W. Hoesler, E. Ritter, and G. Binnig, 1986, Phys. Rev. Lett. 56, 228.

Binnig, G., K. H. Frank, H. Fuchs, N. García, B. Reihl, H. Rohrer, F. Salvan, and A. R. Williams, 1985, Phys. Rev. Lett. 55, 991.

Binnig, G., H. Fuchs, and E. Stoll, 1986, Surf. Sci. 169, L295.

Binnig, G. K., and H. E. Hoenig, 1978, Z. Phys. B 32, 23.

Binnig, G., C. F. Quate, and Ch. Gerber, 1986, Phys. Rev. Lett. 56, 930.

Binnig, G., and H. Rohrer, 1982, Helv. Phys. Acta 55, 726.

Binnig, G., and H. Rohrer, 1983, Surf. Sci. 126, 236.

Binnig, G., and H. Rohrer, 1984, in Proceedings of the 6th General Conference of the European Physical Society, Trends in Physics, edited by J. Janta and J. Pantoflicek (European Physical Society, Prague), Vol. 1, p. 38.

Binnig, G., and H. Rohrer, 1985, Sci. Am. 253, 50.

Binnig, G., and H. Rohrer, 1986, IBM J. Res. Dev. 30, 355.

Binnig, G., H. Rohrer, Ch. Gerber, and E. Stoll, 1984, Surf. Sci. 144, 321.

Binnig, G., H. Rohrer, Ch. Gerber, and E. Weibel, 1982a, Physica B 109\&110, 2075.

Binnig, G., H. Rohrer, Ch. Gerber, and E. Weibel, 1982b, Phys. Rev. Lett. 49, 57.

Binnig, G., H. Rohrer, Ch. Gerber, and E. Weibel, 1983a, Surf. Sci. 131, L379.

Binnig, G., H. Rohrer, Ch. Gerber, and E. Weibel, 1983b, Phys. Rev. Lett. 50, 120.

Bryant, A., D. P. E. Smith, and C. F. Quate, 1986, Appl. Phys. Lett. 48, 832 .

Coleman, R. V., B. Drake, P. K. Hansma, and G. Slough, 1985 , Phys. Rev. Lett. 55, 394.

de Lozanne, A. L., S. A. Elrod, and C. F. Quate, 1985, Phys. Rev. Lett. 54, 2433.

Dordick, Rowan L., 1986, IBM Res. Mag. 24, 2.

Dürig, U., J. K. Gimzewski, and D. W. Pohl, 1986, Phys. Rev. Lett. 57, 2403.

Elrod, S. A., A. Bryant, A. L. de Lozanne, S. Park, D. Smith, and C. F. Quate, 1986, IBM J. Res. Dev. 30, 387.

Ernst, N., and G. Ehrlich, 1986, in Microscopic Methods in Metals, edited by U. Gonser, Topics in Current Physics No. 40 (Springer, Berlin), p. 75.

Feenstra, R. M., and A. P. Fein, 1985, Phys. Rev. B 32, 1394.

Feynman, R. P., 1960, Eng. and Sci. 22.

Fink, H.-W., 1986, IBM J. Res. Dev. 30, 460.

Garcia, N., C. Ocal, and F. Flores, 1983, Phys. Rev. Lett. 50, 2002.

García, R., J. J. Saenz, and N. García, 1986, Phys. Rev. B 33, 4439.

Gatos, Harry C., 1986, Ed., Proceedings of the First International Conference on Scanning Tunneling Microscopy, Santiago de Compostela, Spain [Surf. Sci. 181, Nos. 1 and 2 (1987)].

Gerber, Ch., G. Binnig, H. Fuchs, O. Marti, and H. Rohrer,
1986, Rev. Sci. Instrum. 57, 221.

Giaever, I., 1974, Rev. Mod. Phys. 46, 245.

Golovchenko, J. A., 1986, Science 232, 48.

Guenther, K. H., P. G. Wierer, and J. M. Bennett, 1984, Appl. Opt. 23, 3820.

Hameroff, S., C. Schneiker, A. Scott, P. Jablonka, T. Hensen, D. Sarid, and S. Bell, 1987, unpublished.

Hamers, R. J., R. M. Tromp, and J. E. Demuth, 1986, Phys. Rev. Lett. 56, 1972.

Hansma, P. K., 1985, Bull. Am. Phys. Soc. 30, 251.

Hansma, P. K., and J. Tersoff, 1987, J. Appl. Phys. 61, R1.

Harrison, W. A., 1976, Surf. Sci. 55, 1.

IBM Europe, 1986, Proceedings of the STM Workshop in Oberlech, Austria, 1985 (IBM J. Res. Dev. 30, Nos. 4 \& 5).

Kuk, Y., and P. J. Silverman, 1986, Appl. Phys. Lett. 48, 1597.

Lang, N. D., 1987, Phys. Rev. Lett. 58, 45.

Marti, O., 1986, Ph.D. thesis No. 8095 (ETH, Zürich).

Marti, O., G. Binnig, H. Rohrer, and H. Salemink, 1987, Surf. Sci. 181, 230.

Miskowsky, N. M., P. H. Cutler, T. E. Feuchtwang, S. J. Shepherd, A. A. Lucas, and T. E. Sullivan, 1980, Appl. Phys. Lett. 37, 189.

Moreland, J., S. Alexander, M. Cox, R. Sonnenfeld, and P. K. Hansma, 1983, Appl. Phys. Lett. 43, 387.

Muralt, P., and D. W. Pohl, 1986, Appl. Phys. Lett. 48, 514.

Ott, H. R., and H. Rohrer, 1981, unpublished.

Poppe, U., 1981, Verh. Dtsch. Phys. Ges. (VI) 16, 476.

Quate, C. F., 1986, Phys. Today 39 (No. 8), 26.

Rohrer, H., 1960, Helv. Phys. Acta 33, 675.

Smith, D. P. E., and G. Binnig, 1986, Rev. Sci. Instrum. 57, 2630

Soler, J. M., A. M. Baró, N. García, and H. Rohrer, 1986, Phys.

Rev. Lett. 57, 444.

Sonnenfeld, R., and P. K. Hansma, 1986, Science 232, 211.

Sonnenfeld, R., and B. C. Schardt, 1986, Appl. Phys. Lett. 49, 1172.

Stoll, E., A. Baratoff, A. Selloni, and P. Carnevali, 1984, J. Phys. C 17, 3073.

Takayanagi, K., Y. Tanishiro, M. Takahashi, and S. Takahashi, 1985, J. Vac. Sci. Technol. A 3, 1502.

Teague, E. C., 1978a, Ph.D. thesis (North Texas State University).

Teague, E. C., 1978b, Bull. Am. Phys. Soc. 23, 290.

Teague, E. C., 1986, J. Res. Nat. Bur. Stand. 91, 171.

Tersoff, J., and D. R. Hamann, 1983, Phys. Rev. Lett. 50, 1998.

Thompson, W. A., and S. F. Hanrahan, 1976, Rev. Sci. Instrum. 47, 1303.

Tromp, R. M., 1985, Surf. Sci. 155, 432.

Tromp, R. M., and E. J. van Loenen, 1985, Surf. Sci. 155, 441.

Vieira, S., 1986, IBM J. Res. Dev. 30, 553.

Williamson, B. P., 1967, Proc. Inst. Mech. Eng. London 182, 21.

Young, R., J. Ward, and F. Scire, 1972, Rev. Sci. Instrum. 43, 999. 


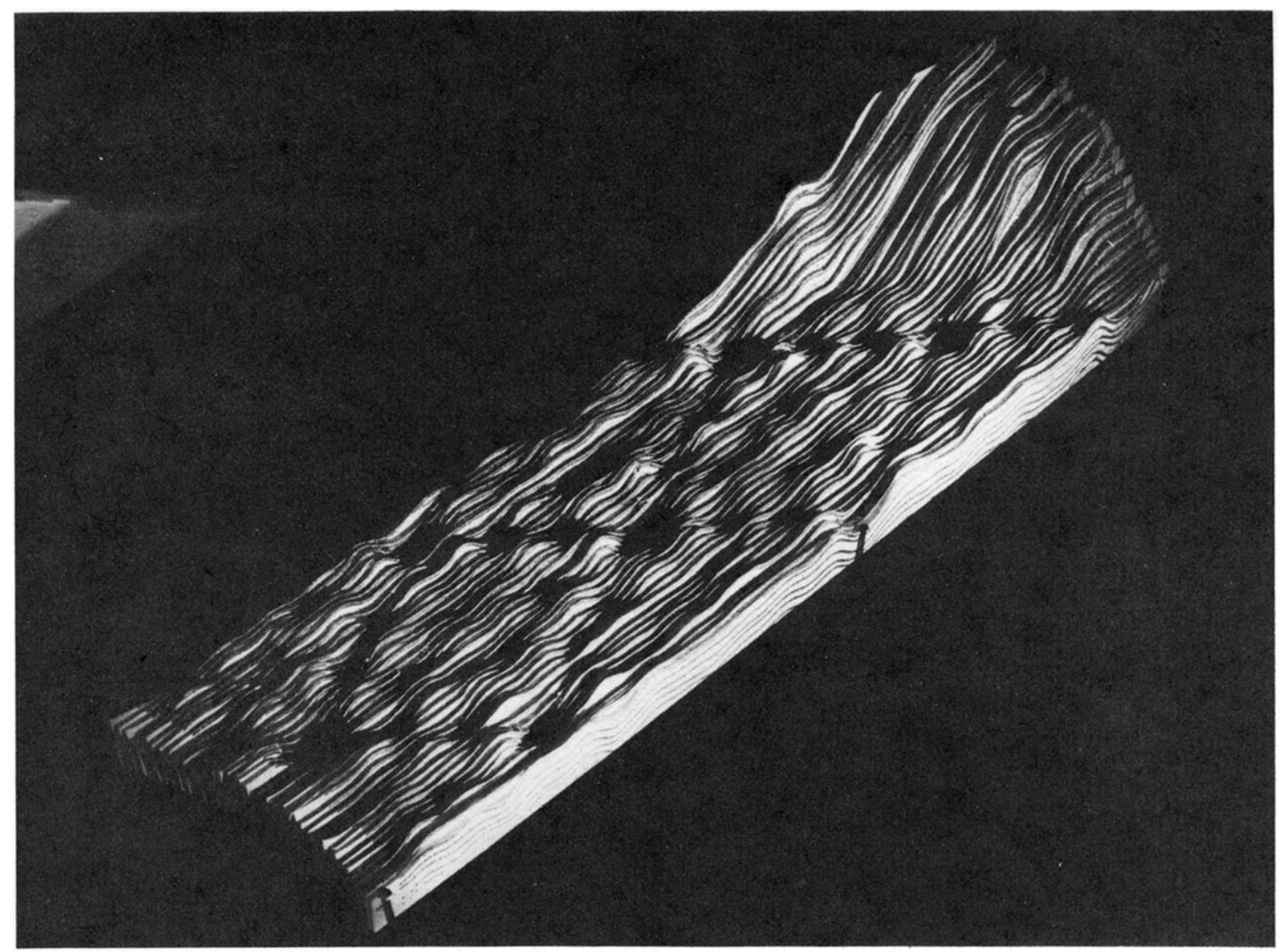

FIG. 6. $7 \times 7$ reconstruction of $\mathrm{Si}(111)$. (a) Relief assembled from the original recorder traces, from Binnig et al. (1983b), (c) 1983 The American Physical Society, and (b) processed image of the $7 \times 7$ reconstruction of $\mathrm{Si}(111)$. Characteristic of the rhombohedral surface unit cell are the corner hole and the 12 maxima, the adatoms. In the processed image, the six adatoms in the right half of the rhombi appear higher. This is an electronic inequivalence on the surface owing to a structural left-right inequivalence in the underlying layers. The reconstruction extends undisturbed to the immediate vicinity of the large "atom hill" on the right. 


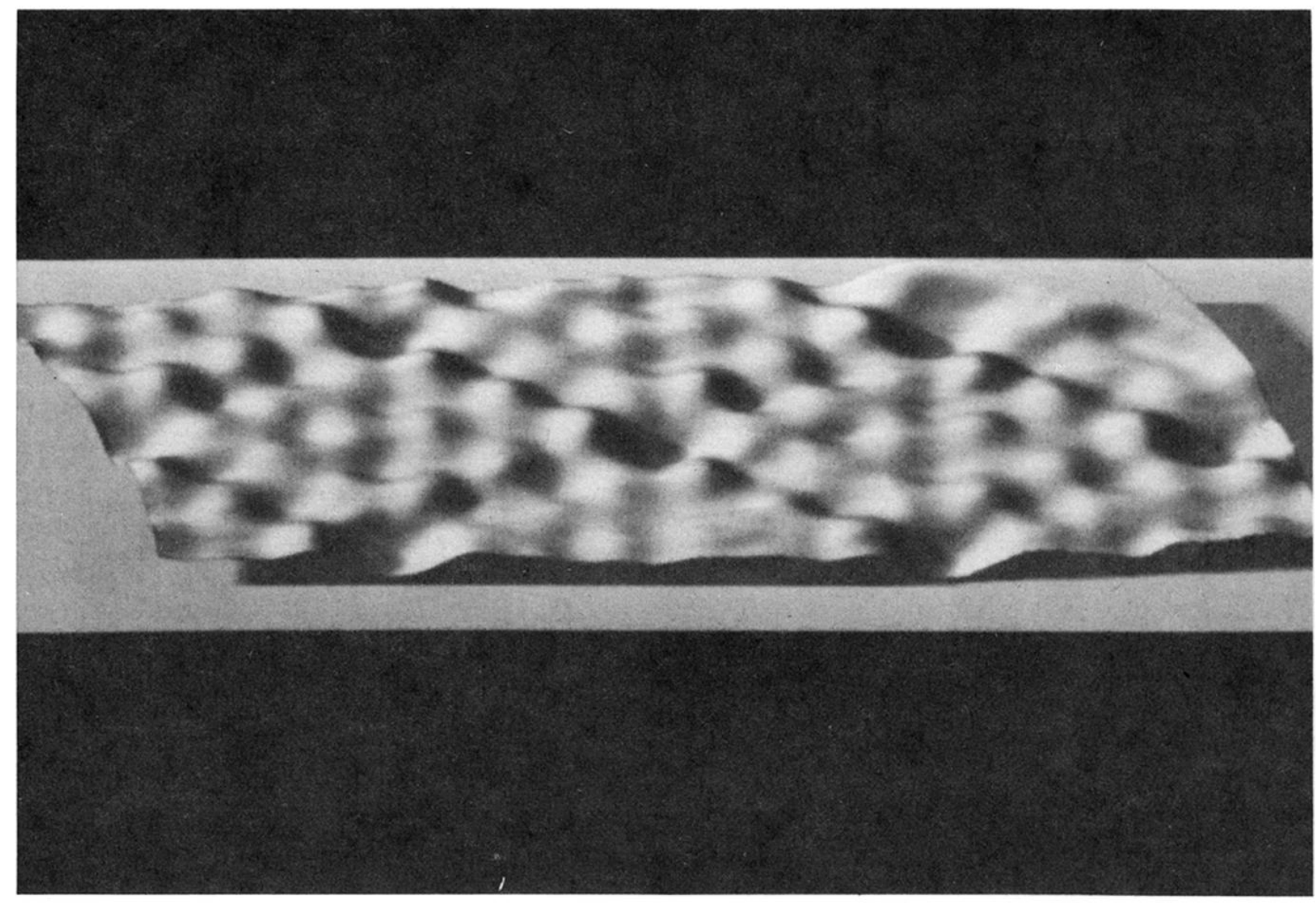

FIG. 6. (Continued).

(b) 


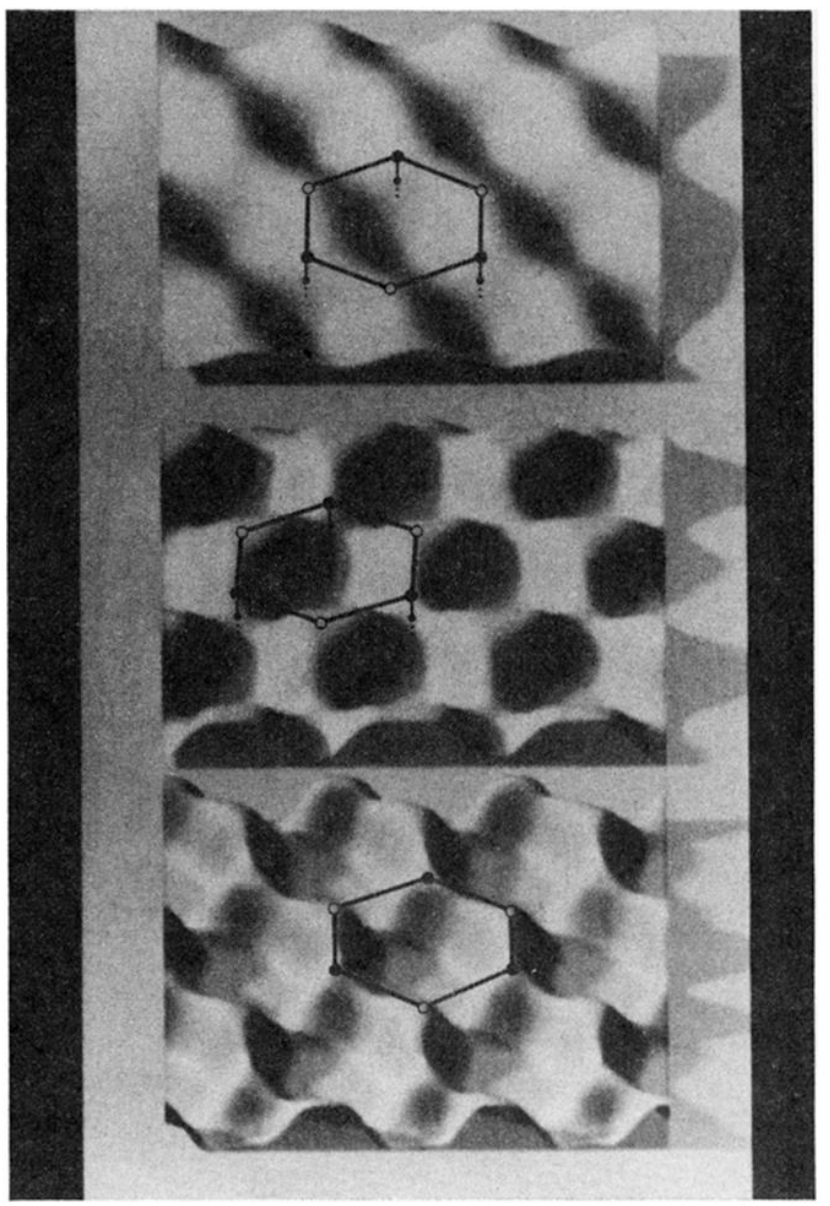

FIG. 7. STM image of cleaved graphite. The top image was taken at a constant tunnel current of $1 \mathrm{nA}$ and at $50 \mathrm{mV}$. The corrugation traced by the tip reflects the local density of states (LDOS) at the Fermi level and not the positions of atoms, which form a flat honeycomb lattice as indicated. The LDOS at the atoms bound to the neighbors in the second layer (open circles) is lower than at the "free" atoms. The image is thus rather a spectroscopic than a topographic one. The middle image is a "current image" showing essentially the same pattern. In the bottom current image, taken closer to the surface, the two inequivalent atoms appear practically identical. This peculiar behavior is compatible with a different local elastic response of the two types of carbon atoms to the interatomic force exerted by the tip compensating for their different LDOS. A local perturbation of the electronic structure might also be important. 


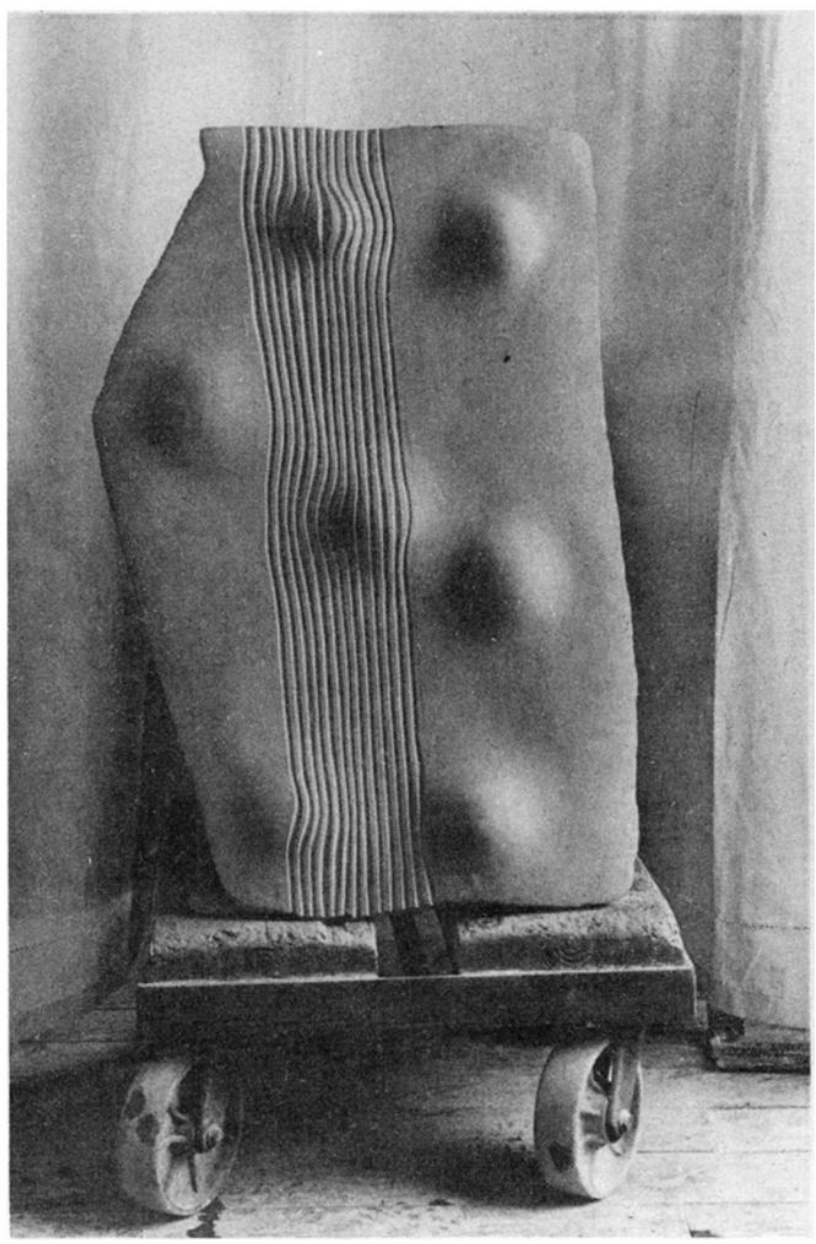

FIG. 8. Artist's conception of spheres. Art and science are both products of the creativity of man, and the beauty of nature is reflected in both. Ruedi Rempfler, the sculptor, found his interpretation in the deformation of a surface. It was the tension of the sphere in its environment which fascinated him, more than the mere portrayal of its shape. An independent creation, its visual and conceptual similarity with Fig. 6 is astounding. Original sculpture by Ruedi Rempfler, photograph courtesy of Thomas P. Frey. 